\title{
Power Indices and Minimal Winning Coalitions in Simple
}

\section{Games in Partition Function Form}

\author{
J.M. Alonso-Meijide ${ }^{1}$, M. Álvarez-Mozos*2, and M.G. Fiestras-Janeiro ${ }^{3}$ \\ ${ }^{1}$ Departamento de Estatística e Investigación Operativa, Facultade de Ciencias, Universidade de \\ Santiago de Compostela (Campus de Lugo). Avda. Alfonxo X O Sabio s/n, 27002, Lugo, Spain. \\ ${ }^{2}$ Departament de Matemàtica Económica, Financera i Actuarial, Facultat d'Economia i Empresa, \\ Universitat de Barcelona. Avda. Diagonal 690, 08034, Barcelona, Spain. \\ ${ }^{3}$ Departamento de Estatística e Investigación Operativa, Facultade de Ciencias Económicas e \\ Empresariais, Universidade de Vigo. Campus Lagoas-Marcosende, 36310, Vigo (Pontevedra), Spain.
}

\begin{abstract}
We propose a generalization of simple games to partition function form games based on a monotonicity property that we define in this context. This property allows us to properly speak about minimal winning embedded coalitions. We propose and characterize two power indices based on this kind of coalitions. Finally, the new indices are used to study the distribution of power in the Parliament of Andalusia emerged after the elections of March 22, 2015.
\end{abstract}

Keywords:

Deegan-Packel index; Public Good index; Simple games; Partition function form; Monotonicity

\section{Introduction}

Game theory provides valuable tools to study how power is distributed among the members of a decision making body. Most of the literature identifies the power of an agent with her ability 
to change the outcome of a ballot. The Shapley-Shubik index (Shapley and Shubik, 1954) is probably the most well known and widely accepted way to measure power. However, many different power indices have been proposed in the literature so far. The Banzhaf index (Banzhaf, 1964) for instance, can be regarded as the non-efficient relative of the Shapley-Shubik index. Indeed, both of them are probabilistic values (Weber, 1988) based on marginal contributions of an agent to coalitions of other agents. In the literature, there are also power indices based on minimal winning coalitions. A minimal winning coalition is a coalition that can pass a bill but where the participation of every member is necessary. That is, it is a winning coalition, which is minimal with respect to inclusion. Deegan and Packel (1978) proposed a power index based on the assumption that all minimal winning coalitions are equally likely and that members of a given minimal winning coalition are equally important. Some years latter, Holler (1982) introduced the Public Good index assuming that the source of power of an agent is the number of minimal winning coalitions in which she participates. Power indices based on different assumptions can be found in Johnston (1978); Alonso-Meijide and Freixas (2010); Álvarez-Mozos et al. (2015). ${ }^{1}$

Most of the mechanisms of decision making bodies are based on voting. Usually, every agent has certain weight and a proposal is approved when the overall weight of the supporters exceeds a given quota. Most of the times, the quota is fixed at simple majority. The classic way to model these processes is by means of simple games in characteristic function form. These games constitute an important subclass of cooperative games in which the worth of every coalition is either 0 (losing) or 1 (winning), the grand coalition is winning, and if a coalition grows then, its worth cannot decrease.

However, we argue that there are other voting mechanisms in legislatures which fall out of the scope of games in characteristic function. In Spain for instance, the election of the President is a duty of the Parliament. Then, if no candidate is supported by a majority of the members in the chamber, minority governments may emerge. The reason behind the formation of such governments is that the rest of the deputies do not agree in favor of an alternative candidate. We believe that these kind of procedures are better modeled by a plurality rule. According to this rule, the winner is the candidate who pools more votes of members of the Parliament than any other candidate. In Section 4 we consider the current Parliament of Andalusia and study winning coalitions with the plurality rule. In case two candidates are supported by the same number of members, we use the votes of citizens as a tie-breaking rule.

Parallel to how games in characteristic function form are appropriate to model the majority

\footnotetext{
${ }^{1}$ We refer to Alonso-Meijide et al. (2013) for a review in the topic.
} 
rule, games in partition function form (Thrall and Lucas, 1963) may be used to describe the plurality rule. In a game in partition function form, the worth of a coalition depends on the arrangement of the rest of agents in coalitions, namely on the whole coalition structure. Therefore, the relevant objects are not coalitions but embedded coalitions that consist of a coalition structure and an active coalition whose worth is being evaluated. Myerson (1977) proposed and characterized an extension of the Shapley value for games in partition function form. Recently, the topic has attracted the attention of researchers and important contributions have been made. For instance, Hafalir (2007) has generalized the concepts of superadditivity and convexity. However, most of the recent contributions deal with generalizations of the Shapley value (see for instance Dutta et al., 2010, and the references therein). In Álvarez-Mozos and Tejada (2015) the Banzhaf value is generalized to games in partition function form.

The literature on simple games in partition function form is quite limited. However, there have been some contributions that are worth mentioning. Bolger $(1983,1990)$ studied different generalizations of the Shapley-Shubik and Banzhaf indices for multi-candidate voting games. These games are $\{0,1\}$-valued but no monotonicity condition is imposed on them. To the best of our knowledge, the only explicit definition of monotonicity for games in partition function form was proposed by Bolger (1986). This property states that if the active coalition grows, while the rest of the agents maintain their coalitional behavior, the worth of the embedded coalition should not decrease. More recently Álvarez-Mozos and Tejada (2015) have proposed the so called ordinal Banzhaf index for this family of simple games in partition function form. Note that, in order to generalize the classic monotonicity property of simple games one just needs to define what growing means for an embedded coalition. Indeed, Myerson (1977) defined a binary relation over the set of embedded coalitions that has been thoroughly studied in Grabisch (2010). From this binary relation, we can infer that an embedded coalition grows when the coalitions, either active or inactive, become larger.

In this paper, we propose a new class of simple games in partition function form. Its main novelty being the monotonicity property that we consider. We assume that an embedded coalition is larger when the active coalition grows while the inactive coalitions become more divided. The plurality rule described above fits this idea of growth. We would like to point out that the same idea was used in a different setting by Carreras and Magaña (2008). However, to the best of our knowledge, it is the first time in which this family of games is studied.

A way to develop power indices for the new family of simple games is to apply values defined for games in partition function form. This is the object of Álvarez-Mozos et al. (2016), where 
a generalization of the Shapley-Shubik index is characterized. In this paper we take a different approach and generalize the Deegan-Packel and Public Good indices. Moreover, we also provide two characterizations for each index by means of four properties. As we will see, the basic properties of efficiency, null player, and symmetry (Felsenthal and Machover, 1998) are easily generalized to our class of games. Then, in order to single out each of the proposed indices we can use either a merging property (Deegan and Packel, 1978; Holler and Packel, 1983) or a monotonicity property (Lorenzo-Freire et al., 2007; Alonso-Meijide et al., 2008).

The rest of the paper is organized as follows. In Section 2 we introduce the key notion of inclusion. This allows us to define monotonic games in partition function form and minimal winning embedded coalitions. In Section 3, we introduce the DP and PG power indices. Then, we provide two characterizations of each of them by means of four properties. Finally, in Section 4 a real example from the political field is used to illustrate the performance of our indices.

\section{Simple games in partition function form}

Let $N$ be a finite set of players, then the set of partitions of $N$ is denoted by $\mathcal{P}(N)$. For convenience, we assume that the empty set is an element of every partition, i.e., for every $P \in \mathcal{P}(N), \emptyset \in P$. An embedded coalition of $N$ is a pair $(S, P)$ where $P \in \mathcal{P}(N)$ and $S \in P$. We will refer to $S$ as the active coalition in $P$. The set of embedded coalitions of $N$ is denoted by $E C^{N}$, i.e. $E C^{N}=\{(S, P): P \in \mathcal{P}(N)$ and $S \in P\}$. We may abuse language and say that a player $i \in N$ participates in an embedded coalition $(S, P) \in E C^{N}$ if player $i$ belongs to $S$. We simplify and write $S \cup i$ and $S \backslash i$ instead $S \cup\{i\}$ and $S \backslash\{i\}$, respectively. Given $P \in \mathcal{P}(N)$ and a nonempty coalition $S \in P$, we let $P_{-S} \in \mathcal{P}(N \backslash S)$ denote the partition $P \backslash\{S\}$. Lastly, given $P \in \mathcal{P}(N)$ and $i \in N$, we denote by $P(i)$ the element of $P$ that contains $i$, i.e., $P(i) \in P$ such that $i \in P(i)$.

A game in partition function form is a pair $(N, v)$, where $N$ is the finite set of players and $v$ is the partition function that assigns to every embedded coalition a real number, i.e., $v: E C^{N} \rightarrow \mathbb{R}$, with the convention that for every $P \in \mathcal{P}(N), v(\emptyset, P)=0$. The real number $v(S, P)$ is to be understood as the worth of coalition $S$ when the players are organized according to $P$. The set of games in partition function form with common player set $N$ is denoted by $\mathcal{G}^{N}$ and the set of games in partition function form with an arbitrary player set is denoted by $\mathcal{G}$. It is easy to notice that $\mathcal{G}^{N}$ is a vector space over $\mathbb{R}$. Indeed, de Clippel and Serrano (2008) devised a basis of the vector space that generalizes the basis of games in characteristic function 
that consists of unanimity games. Given $(S, P) \in E C^{N}$, with $S \neq \emptyset$, let $\left(N, e_{(S, P)}\right) \in \mathcal{G}$ be defined for every $(T, Q) \in E C^{N}$ by

$$
e_{(S, P)}(T, Q)= \begin{cases}1 & \text { if } S \subseteq T \text { and } \forall T^{\prime} \in Q_{-T}, \exists S^{\prime} \in P \text { such that } T^{\prime} \subseteq S^{\prime} \\ 0 & \text { otherwise. }\end{cases}
$$

Then, de Clippel and Serrano (2008) show that $\left\{\left(N, e_{(S, P)}\right):(S, P) \in E C^{N}\right.$ and $\left.S \neq \emptyset\right\}$ constitutes a basis of $\mathcal{G}^{N}$.

In this paper we are concerned with a subclass of $\mathcal{G}$ that generalizes simple games in characteristic function form as introduced by von Neumann and Morgenstern (1944). For doing so, we develop a concept of monotonicity for games in partition function form. The intuition behind monotonic games is that the enlargement of a coalition cannot cause a decrease in its worth. Therefore, in order to generalize this idea, we use a notion of inclusion for embedded coalitions that will be of key importance for our results and that it is implicitly formulated in Eq. (1).

Definition 2.1. Let $N$ be a finite set and $(S, P),(T, Q) \in E C^{N}$. We define the inclusion among embedded coalitions as follows:

$$
(S, P) \subseteq(T, Q) \Longleftrightarrow S \subseteq T \text { and } \forall T^{\prime} \in Q_{-T}, \exists S^{\prime} \in P \text { such that } T^{\prime} \subseteq S^{\prime}
$$

Note that whenever $S \neq \emptyset,(S, P) \subseteq(T, Q)$ if and only if $e_{(S, P)}(T, Q)=1$. According to the above definition, an embedded coalition $(S, P)$ is a subset of another embedded coalition $(T, Q)$ if the coalition $S$ is a subset of $T$ and moreover, the partition $\{R \backslash T: R \in P\}$ is coarser than $Q_{-T}$. Notice that both families of coalitions are partitions of $N \backslash T$.

We are now in the position to introduce the class of games under study.

Definition 2.2. A game in partition function form $(N, v) \in \mathcal{G}$ is said to be a simple game in partition function form if it satisfies the three conditions below:

i) For every $(S, P) \in E C^{N}, v(S, P) \in\{0,1\}$.

ii) $v(N,\{\emptyset, N\})=1$.

iii) If $(S, P),(T, Q) \in E C^{N}$ such that $(S, P) \subseteq(T, Q)$, then $v(S, P) \leq v(T, Q)$.

An embedded coalition, $(S, P) \in E C^{N}$, is said to be winning if $v(S, P)=1$ and losing otherwise. A game in partition function form satisfying condition iii) is said to be monotonic. The set of simple games in partition function form with common player set $N$ is denoted by $\mathcal{S G}^{N}$ and the set of simple games in partition function form with an arbitrary player set is denoted by $\mathcal{S G}$. 
Simple games in partition function form can model parliamentary procedures based on the plurality rule. First, each embedded coalition is either winning or losing. Second, the grand coalition is always winning. ${ }^{2}$ Third, suppose that $(S, P) \in E C^{N}$ is a winning embedded coalition, then if coalition $S$ grows or the remaining agents are more divided the resulting embedded coalition remains to be winning.

It is straightforward to check that the games that form the basis of de Clippel and Serrano (2008), see Eq. (1), are instances of simple games in partition function form as defined above. That is, if $N$ is a finite set of players, then for every $(S, P) \in E C^{N},\left(N, e_{(S, P)}\right) \in \mathcal{S G}$.

The monotonicity property considered above allows us to properly speak about minimal winning embedded coalitions. Let $(N, v) \in \mathcal{S G}$. A winning embedded coalition $(S, P) \in E C^{N}$ is said to be minimal if every proper subset of it is a losing embedded coalition, i.e., if $(T, Q) \subsetneq$ $(S, P)$ implies that $v(T, Q)=0 .{ }^{3}$ The set of all minimal winning embedded coalitions of a simple game in partition function form is denoted by $\mathcal{M}(v)$ and the subset of minimal winning embedded coalitions that contain a given player $i \in N$ is denoted by $\mathcal{M}_{i}(v)$, i.e., $\mathcal{M}_{i}(v)=$ $\{(S, P) \in \mathcal{M}(v): i \in S\}$.

A player $i \in N$ is said to be a null player in $(N, v) \in \mathcal{S G}$ if she does not participate in any minimal winning embedded coalition, i.e., $\mathcal{M}_{i}(v)=\emptyset$. Similarly, two players $i, j \in N$ are said to be symmetric in $(N, v) \in \mathcal{S G}$ if exchanging the two players does not change the worth of an embedded coalition, i.e., if for every $(S, P) \in E C^{N}$ such that $S \subseteq N \backslash\{i, j\}$,

$$
\left(S \cup i, P_{-S, P(i)} \cup\{S \cup i, P(i) \backslash i\}\right) \in \mathcal{M}(v) \Leftrightarrow\left(S \cup j, P_{-S, P(j)} \cup\{S \cup j, P(j) \backslash j\}\right) \in \mathcal{M}(v),
$$

where $P_{-S, P(k)}=\left(P_{-S}\right)_{-P(k)}$, for every $k \in\{i, j\}$.

It is easy to notice, that as a consequence of the monotonicity property, a simple game in partition function form is completely determined by the set of minimal winning embedded coalitions of the game. In a sense, all the relevant information of a simple game in partition function form is condensed in the set of minimal winning embedded coalitions. We state this fact formally in the two propositions below.

Proposition 2.1. Every simple game in partition function form can be obtained as the maximum of games in a subset of $\left\{\left(N, e_{(S, P)}\right):(S, P) \in E C^{N}\right.$ and $\left.S \neq \emptyset\right\}$. Indeed, for every $(N, v) \in$ $\mathcal{S G}$ and every $(T, Q) \in E C^{N}$ it holds that

$$
v(T, Q)=\max \left\{e_{(S, P)}(T, Q):(S, P) \in \mathcal{M}(v)\right\} .
$$

\footnotetext{
${ }^{2}$ We can talk about the grand coalition because there is a single embedded coalition of the type $(N, P)$.

${ }^{3}$ A proper subset, $(T, Q) \subsetneq(S, P)$, is a subset $(T, Q) \subseteq(S, P)$ satisfying $(T, Q) \neq(S, P)$.
} 
Proof. Let $(N, v) \in \mathcal{S G}$ and $(T, Q) \in E C^{N}$. On the one hand, if $(T, Q)$ is a winning embedded coalition then, by definition there is $(S, P) \subseteq(T, Q)$ where $(S, P) \in \mathcal{M}(v)$ and $v(T, Q)=\max \left\{e_{(S, P)}(T, Q):(S, P) \in \mathcal{M}(v)\right\}=1$. On the other hand, if $(T, Q)$ is a losing embedded coalition then, since $(N, v)$ is monotonic it holds that for every $(S, P) \subseteq(T, Q)$, $(S, P)$ is a losing embedded coalition. In particular there is no minimal winning embedded coalition contained in $(T, Q)$ and thus, $v(T, Q)=\max \left\{e_{(S, P)}(T, Q):(S, P) \in \mathcal{M}(v)\right\}=0$.

Proposition 2.2. Let $\mathcal{C} \subseteq E C^{N}$ be such that for every $(S, P),(T, Q) \in \mathcal{C}$ with $(S, P) \neq(T, Q)$, $(S, P) \nsubseteq(T, Q)$ and $(T, Q) \nsubseteq(S, P)$. Then, there exists a unique simple game in partition function form, $(N, v)$, such that $\mathcal{M}(v)=\mathcal{C}$.

Proof. Let $\mathcal{C} \subseteq E C^{N}$ be such that for every $(S, P),(T, Q) \in \mathcal{C}$ with $(S, P) \neq(T, Q)$, $(S, P) \nsubseteq(T, Q)$ and $(T, Q) \nsubseteq(S, P)$. Define $(N, v) \in \mathcal{G}$ for every $(T, Q) \in E C^{N}$ by $v(T, Q)=$ $\max \left\{e_{(S, P)}(T, Q):(S, P) \in \mathcal{C}\right\}$. It is easy to see that $(N, v) \in \mathcal{S G}$. Since there is no inclusion relation among the embedded coalitions in $\mathcal{C}$, it follows that $\mathcal{M}(v)=\mathcal{C}$. Finally, we show that such $(N, v)$ is unique. Indeed, suppose $(N, w) \in \mathcal{S G}$ satisfying $\mathcal{M}(w)=\mathcal{C}$. Then, by Proposition 2.1, for every $(S, P) \in E C^{N}, w(S, P)=\max \left\{e_{(S, P)}(T, Q):(S, P) \in \mathcal{M}(w)\right\}=$ $\max \left\{e_{(S, P)}(T, Q):(S, P) \in \mathcal{C}\right\}=v(S, P)$, which concludes the proof.

\section{Power indices based on minimal winning embedded coali- tions}

In this section, we first propose natural generalizations of the Deegan-Packel and Public Good indices (see Deegan and Packel, 1978; Holler, 1982) for simple games in partition function form. Next, we consider a number of properties and characterize the proposed indices.

A power index is a mapping, $\mathrm{f}$, that assigns to every simple game $(N, v) \in \mathcal{S G}$ a vector $\mathrm{f}(N, v) \in \mathbb{R}^{N}$, where $\mathrm{f}_{i}(N, v)$ describes the power of agent $i \in N$.

Definition 3.1. DP is the power index defined for every $(N, v) \in \mathcal{S G}$ and $i \in N$ by

$$
\operatorname{DP}_{i}(N, v)=\frac{1}{|\mathcal{M}(v)|} \sum_{(S, P) \in \mathcal{M}_{i}(v)} \frac{1}{|S|}
$$

The motivation behind the above definition emerges from three assumptions on how agents behave in a simple game in partition function form. First, only minimal winning embedded coalitions should be considered. The first assumption is twofold, on the one hand it requires the 
active coalition to be of a minimal size and on the other hand, it demands the inactive coalitions to be of maximal size. Second, every minimal winning embedded coalition emerges with the same probability. Third, the agents participating in a minimal winning embedded coalition share the power equally.

Definition 3.2. PG is the power index defined for every $(N, v) \in \mathcal{S G}$ and $i \in N$ by

$$
\mathrm{PG}_{i}(N, v)=\frac{\left|\mathcal{M}_{i}(v)\right|}{\sum_{j \in N}\left|\mathcal{M}_{j}(v)\right|} .
$$

Similar to DP, the definition of PG above considers that in order to measure the power of an agent in a simple game in partition function form only minimal winning embedded coalitions should be taken into consideration. The main difference lies in the fact that PG is not sensitive to the sizes of minimal winning embedded coalitions. Instead, it suggests that the power of an agent stems from the number of minimal winning embedded coalitions in which she participates.

Next, we describe a number of properties that a power index may satisfy. The first three are reformulations of standard axioms in cooperative game theory.

EFF A power index $f$ satisfies efficiency if for every $(N, v) \in \mathcal{S G}$,

$$
\sum_{i \in N} \mathrm{f}_{i}(N, v)=1 .
$$

NPP A power index $\mathrm{f}$ satisfies the null player property if for every $(N, v) \in \mathcal{S G}$ and every null player $i \in N$ in $(N, v)$,

$$
\mathrm{f}_{i}(N, v)=0 .
$$

SYM A power index $\mathrm{f}$ satisfies symmetry if for every $(N, v) \in \mathcal{S G}$ and every pair $i, j \in N$ of symmetric players in $(N, v)$,

$$
\mathrm{f}_{i}(N, v)=\mathrm{f}_{j}(N, v) .
$$

Little discussion is needed on the three properties above. Indeed, they are trivial adaptations of properties that any sensible power index should satisfy (Felsenthal and Machover, 1998).

In order to single out the power indices DP or PG we need an additional property. As we will see, the two merging properties introduced in Deegan and Packel (1978) and Holler and Packel (1983) can be easily adapted to our setting of games in partition function form. The common feature of the aforementioned properties is that they require the power in the maximum of certain pairs of simple games to be weighted averages of the powers in the two 
games. Obviously, the two properties only differ on the weights used in the average. Before we can present these adaptations we have to introduce some additional concepts. For every $(N, v),(N, w) \in \mathcal{S G}$, we define the maximum game, $(N, v \vee w) \in \mathcal{S G}$, for every $(S, P) \in E C^{N}$ by $(v \vee w)(S, P)=\max \{v(S, P), w(S, P)\}$. It is easy to notice that a minimal winning embedded coalition of the maximum game is a minimal winning embedded coalition in one of the two original games. However, there are cases in which minimal winning embedded coalitions in the original games are not so in the maximum game. These situations are ruled out in the following concept. Two simple games in partition function form $(N, v),(N, w) \in \mathcal{S G}$ are said to be mergeable if

$$
\forall(S, P) \in \mathcal{M}(v) \text { and }(T, Q) \in \mathcal{M}(w), \quad(S, P) \nsubseteq(T, Q) \text { and }(T, Q) \nsubseteq(S, P)
$$

It is straightforward to check that the minimal winning embedded coalitions in the maximum of two mergeable games is precisely the union of the minimal winning embedded coalitions in the original simple games in partition function form. The two properties below describe the distribution of power in the maximum of two mergeable simple games in partition function form.

DP-MER A power index $\mathrm{f}$ satisfies DP-mergeability if for every pair of mergeable simple games in partition function form and every $i \in N,(N, v),(N, w) \in \mathcal{S G}$,

$$
\mathrm{f}_{i}(N, v \vee w)=\frac{|\mathcal{M}(v)| \cdot \mathrm{f}_{i}(N, v)+|\mathcal{M}(w)| \cdot \mathrm{f}_{i}(N, w)}{|\mathcal{M}(v \vee w)|} .
$$

PG-MER A power index $\mathrm{f}$ satisfies $P G$-mergeability if for every pair of mergeable simple games in partition function form $(N, v),(N, w) \in \mathcal{S G}$ and every $i \in N$,

$$
\mathrm{f}_{i}(N, v \vee w)=\frac{\sum_{j \in N}\left|\mathcal{M}_{j}(v)\right| \cdot \mathrm{f}_{i}(N, v)+\sum_{j \in N}\left|\mathcal{M}_{j}(w)\right| \cdot \mathrm{f}_{i}(N, w)}{\sum_{j \in N}\left|\mathcal{M}_{j}(v \vee w)\right|} .
$$

Both DP-MER and PG-MER state that the power in the maximum of two mergeable simple games in partition function form is a weighted average of the power in each of the original games. According to DP-MER the weights are given by the ratio of the number of minimal winning embedded coalitions in the original game and in the maximum game. Meanwhile, PGMER uses weights given by the ratio of the sum of cardinalities of minimal winning embedded coalitions in which every agent participates both in the original game and in the maximum game. 
Theorem 3.1. DP is the only power index satisfying EFF, NPP, SYM, and DP-MER.

Proof. Using the definition, it is straightforward to check that DP satisfies EFF, NPP, and SYM. To see that it also satisfies DP-MER, it is enough to take into account that for every pair of mergeable simple games in partition function form, $(N, v),(N, w) \in \mathcal{S G}$, it holds that $\mathcal{M}(v \vee w)=\mathcal{M}(v) \cup \mathcal{M}(w)$.

Next, we show that DP is indeed the only power index satisfying EFF, NPP, SYM, and DP-MER. We proceed by induction on the number of minimal winning embedded coalitions. Let $f$ be a power index satisfying the four properties. First, let $(N, v) \in \mathcal{S G}$ be such that $|\mathcal{M}(v)|=1$. Then, $\mathcal{M}(v)=\{(S, P)\}$ for some $(S, P) \in E C^{N}$ and $v=e_{(S, P)}$. It is immediate to check that every $i \notin S$ is a null player in $\left(N, e_{(S, P)}\right)$. Then, by $\mathrm{NPP}, \mathrm{f}_{i}\left(N, e_{(S, P)}\right)=0$. Similarly, every pair of players in $S$ are symmetric in $\left(N, e_{(S, P)}\right)$. Then, by SYM they get the same payoff and by EFF we conclude that for every $i \in S, \mathrm{f}_{i}\left(N, e_{(S, P)}\right)=\frac{1}{|S|}$. Second, suppose that $\mathrm{f}$ is uniquely determined for every $(N, v) \in \mathcal{S G}$ with $|\mathcal{M}(v)|<r$. Let $(N, v) \in \mathcal{S G}$ with $\mathcal{M}(v)=\left\{\left(S_{1}, P^{1}\right), \ldots,\left(S_{r}, P^{r}\right)\right\}$. Next, by Proposition 2.1 we have that for every $(T, Q) \in E C^{N}$,

$$
v(T, Q)=\max \left\{e_{(S, P)}(T, Q):(S, P) \in \mathcal{M}(v)\right\}=\max \left\{w(T, Q), e_{\left(S_{r}, P^{r}\right)}(T, Q)\right\}
$$

where $w(T, Q)=\max \left\{e_{\left(S_{k}, P^{k}\right)}(T, Q): k \in\{1, \ldots, r-1\}\right\}$. Since $\left\{\left(S_{1}, P^{1}\right), \ldots,\left(S_{r}, P^{r}\right)\right\}$ is the set of all minimal winning embedded coalitions of $(N, v)$ then, for every $k \in\{1, \ldots, r-1\}$, $\left(S_{k}, P^{k}\right) \nsubseteq\left(S_{r}, P^{r}\right)$ and $\left(S_{r}, P^{r}\right) \nsubseteq\left(S_{k}, P^{k}\right)$. Then, $(N, w)$ and $\left(N, e_{\left(S_{r}, P^{r}\right)}\right)$ are mergeable games and by DP-MER for every $i \in N$,

$$
\mathrm{f}_{i}(N, v)=\mathrm{f}_{i}\left(N, w \vee e_{\left(S_{r}, P^{r}\right)}\right)=\frac{(r-1) \cdot \mathrm{f}_{i}(N, w)+\mathrm{f}_{i}\left(N, e_{\left(S_{r}, P^{r}\right)}\right)}{r} .
$$

Finally, the two payoffs in the right hand side of the equation above are uniquely determined by the induction hypothesis and the proof concludes.

Theorem 3.2. PG is the only power index satisfying EFF, NPP, SYM, and PG-MER.

Proof. It is straightforward to check that PG satisfies EFF, NPP, and SYM. By definition, for every $(N, v) \in \mathcal{S G}$ and and $i \in N, \mathrm{PG}_{i}(N, v) \sum_{j \in N}\left|\mathcal{M}_{j}(v)\right|=\left|\mathcal{M}_{i}(v)\right|$ and it follows that PG satisfies PG-MER. The uniqueness part follows the same lines as we did in the proof of Theorem 3.1. The only difference is in the last step where instead of DP-MER we now apply PG-MER and instead of Eq. (2) we obtain

$$
\mathrm{f}_{i}\left(N, w \vee e_{\left(S_{r}, P^{r}\right)}\right)=\frac{\sum_{j \in N}\left|\mathcal{M}_{j}(w)\right| \cdot \mathrm{f}_{i}(N, w)+\sum_{j \in N}\left|\mathcal{M}_{j}\right|\left(e_{\left(S_{r}, P^{r}\right)}\right) \mid \cdot \mathrm{f}_{i}\left(N, e_{\left(S_{r}, P^{r}\right)}\right)}{\sum_{j \in N}\left|\mathcal{M}_{j}(v)\right|},
$$


for every $i \in N$. As in the proof of Theorem 3.1, the uniqueness follows by induction.

Next, we introduce two monotonicity properties that give rise to two alternative characterizations of DP and PG. Monotonicity properties describe how the payoff of a given player changes when her position in the game is strengthened. Lorenzo-Freire et al. (2007) and Alonso-Meijide et al. (2008) introduced two monotonicity properties in the context of games in characteristic function that can be easily adapted to games in partition function form. Indeed, consider the following two properties that a power index can be asked to satisfy.

DP-MON A power index $\mathrm{f}$ satisfies DP-monotonicity if for every pair of simple games in partition function form, $(N, v),(N, w) \in \mathcal{S G}$ and every $i \in N$ with $\mathcal{M}_{i}(v) \subseteq \mathcal{M}_{i}(w)$ we have

$$
\mathrm{f}_{i}(N, w)|\mathcal{M}(w)| \geq \mathrm{f}_{i}(N, v)|\mathcal{M}(v)| .
$$

PG-MON A power index $\mathrm{f}$ satisfies $P G$-monotonicity if for every pair of simple games in partition function form, $(N, v),(N, w) \in \mathcal{S G}$ and every $i \in N$ with $\mathcal{M}_{i}(v) \subseteq \mathcal{M}_{i}(w)$ we have

$$
\mathrm{f}_{i}(N, w) \sum_{j \in N}\left|\mathcal{M}_{j}(w)\right| \geq \mathrm{f}_{i}(N, v) \sum_{j \in N}\left|\mathcal{M}_{j}(v)\right| .
$$

Both DP-MON and PG-MON state that when a player participates in more minimal winning embedded coalitions her power scaled by a number increases. According to DP-MON, the power times the number of minimal winning embedded coalitions increases. On the other hand, PGMON states that the power times the sum of cardinalities of minimal winning embedded coalitions in which every agent participates increases. Note that if the set of minimal winning embedded coalitions in which player $i$ participates is the same in both games, these properties give a direct relation between the power of $i$ in the two games.

Theorem 3.3. DP is the only power index satisfying EFF, NPP, SYM, and DP-MON.

Proof. It is clear that DP satisfies EFF, NPP, and SYM. Let $(N, v),(N, w) \in \mathcal{S G}$ and $i \in N$ with $\mathcal{M}_{i}(v) \subseteq \mathcal{M}_{i}(w)$, we have

$$
\operatorname{DP}_{i}(N, w)|\mathcal{M}(w)|=\sum_{(S, P) \in \mathcal{M}_{i}(w)} \frac{1}{|S|} \geq \sum_{(S, P) \in \mathcal{M}_{i}(v)} \frac{1}{|S|}=\operatorname{DP}_{i}(N, v)|\mathcal{M}(v)|
$$

Then, DP satisfies DP-MON.

Next, we prove the uniqueness by induction on $|\mathcal{M}(v)|$. Let $\mathrm{f}$ be a power index satisfying EFF, NPP, SYM, and DP-MON. Note that the reasoning in the proof of Theorem 3.1 can be repeated for the base case of the induction. Let us assume that the result is true for all $(N, v) \in \mathcal{S G}$ 
with $|\mathcal{M}(v)|<r$, for some $r>1$. Let $(N, v) \in \mathcal{S G}$ such that $\mathcal{M}(v)=\left\{\left(S_{1}, P^{1}\right), \ldots,\left(S_{r}, P^{r}\right)\right\}$ with $r>1$ and $R=S_{1} \cap \cdots \cap S_{r}$. Note that $R \neq N$ because $r>1$. Then, consider $i \notin R$. If $i \notin S_{l}$ for every $l=1, \ldots, r$, then $i$ is a null player and $\mathrm{f}_{i}(N, v)=0$ by NPP. If there is some $l \in\{1, \ldots, r\}$ such that $i \in S_{l} \backslash R$ then, define $(N, w) \in \mathcal{S G}$ with $\mathcal{M}(w)=\mathcal{M}_{i}(v)$. Since the embedded coalitions in $\mathcal{M}_{i}(v)$ are minimal winning in $(N, v)$, there is no inclusion relation among them. Then, by Proposition 2.2, $(N, w) \in \mathcal{S G}$ is unique and well defined. Next, taking into account that $\mathcal{M}_{i}(w)=\mathcal{M}_{i}(v)$ and applying the DP-MON property twice,

$$
\mathrm{f}_{i}(N, v)|\mathcal{M}(v)|=\mathrm{f}_{i}(N, w)|\mathcal{M}(w)| .
$$

Note that since $i \notin R,|\mathcal{M}(w)|<|\mathcal{M}(v)|$. Then, the right hand side of Eq. (3) is unique by induction and so, $\mathrm{f}_{i}(N, v)$ is uniquely determined. Finally, let $i \in R$. By EFF, $\sum_{j \in R} \mathrm{f}_{j}(N, v)$ is uniquely determined. Suppose that $|R|>1$, otherwise the proof concludes. Notice that every pair of players $j, k \in R$ are symmetric in $(N, v) \in \mathcal{S G}$. Then by SYM, $\mathrm{f}_{i}(N, v)$ is also unique.

In a similar way we can characterize the PG power index using the PG-MON property.

Theorem 3.4. PG is the only power index satisfying EFF, NPP, SYM, and PG-MON.

Proof. On the one hand, we already now that PG satisfies EFF, NPP, and SYM. By definition, for every $(N, v) \in \mathcal{S G}$ and and $i \in N, \mathrm{PG}_{i}(N, v) \sum_{j \in N}\left|\mathcal{M}_{j}(v)\right|=\left|\mathcal{M}_{i}(v)\right|$ and it follows that PG satisfies PG-MON. On the other hand, for the uniqueness we follow the same lines as in Theorem 3.3. The only difference is in the last step where instead of DP-MON we now apply PG-MON and instead of Eq. (3) we obtain

$$
\mathrm{f}_{i}(N, v) \sum_{j \in N}\left|\mathcal{M}_{j}(v)\right|=\mathrm{f}_{i}(N, w) \sum_{j \in N}\left|\mathcal{M}_{j}(w)\right|
$$

for $i \notin R$. Thus, the uniqueness follows by induction. The reasoning for $i \in R$ is analogous.

\section{A political example}

In this section, we illustrate the performance of the new power indices and compare the distribution of power in the usual simple game in characteristic function with that of the simple game in partition function. The Parliament of Andalusia, one of Spain's seventeen regions, is constituted by 109 members. Since the elections held in 2015, the Parliament consists of 47 members of the social-democratic party PSOE $(P S), 33$ members of the conservative party PP $(P P), 15$ members of the new left-wing party Podemos $(P O), 9$ members of the new liberal 
party Ciudadanos $(C I)$, and 5 members of the traditional left-wing party IU $(I U)$. After a long negotiation process, the candidate of $P S$ was invested President of the regional government with the votes of $P S$ and $C I$. It is important to point out that we abstract from the ideological and political strategies of the parties. In order to account for these considerations there are more sophisticated models that could be used (see for instance Alonso-Meijide and Bowles, 2005; Alonso-Meijide et al., 2009).

In the first place, we consider the most common decision rule in the Parliament which is the simple majority. More precisely, let $N=\{P S, P P, P O, C I, I U\}$ and $w=\left(w_{P S}, w_{P P}, w_{P O}, w_{C I}, w_{I U}\right)=$ $(47,33,15,9,5)$. Then for every $S \subseteq N, v(S)=1$ if and only if $\sum_{i \in S} w_{i} \geq 55$. In this simple game in characteristic function there are only 4 minimal winning coalitions, namely $\{P S, P P\},\{P S, P O\},\{P S, C I\}$, and $\{P P, P O, C I\}$. From an inspection of the minimal winning coalitions we conclude that $I U$ is a null player and $P P, P O$, and $C I$ are symmetric players.

In the second place, we consider a decision procedure based on the plurality rule. To be precise, we define the simple game in partition function form, $(N, v) \in \mathcal{S G}$, as follows:

i) If there is one coalition $S$ supported by more members than any other coalition in a given coalition structure $P$, i.e., if $\sum_{i \in S} w_{i}>\sum_{i \in T} w_{i}$, for every $T \in P_{-S}$. Then, $v(S, P)=1$ and $v(T, P)=0$ for every $T \in P_{-S}$.

ii) In case there are draws among the coalitions with maximum overall weight we use a tiebreaking rule to determine the winning coalition. For the case of a Parliament, we propose to count the number of votes in the elections. In the example under consideration there is one coalition structure, namely $P=\{\{P S\},\{P P, C I, I U\},\{P O\}\}$ in which the coalitions $\{P S\}$ and $\{P P, C I, I U\}$ are supported by the same number of members. In the elections $P S$ obtained 1409042 votes while the coalition $\{P P, C I, I U\}$ was supported by 1707083 citizens. Therefore, $v(\{P P, C I, I U\}, P)=1, v(\{P S\}, P)=0$, and $v(\{P O\}, P)=0$.

Notice that in the simple game in partition function described above for every coalition structure there is only one winning coalition.

In Table 1 we list the 9 minimal winning embedded coalitions of the simple game in partition function form.

Observe that there are more minimal winning embedded coalitions in the game in partition function form than minimal winning coalitions in the game in characteristic function form. Given the higher complexity of the model, this is a fact that could be expected. Note that $I U$ is not null when we consider the plurality rule because it participates in two minimal winning 


\begin{tabular}{|l|l|}
\hline Active coalition & \multicolumn{1}{|c|}{ Coalition structure } \\
\hline$\{P S\}$ & $\{P S\},\{P P\},\{P O, C I, I U\}$ \\
$\{P S\}$ & $\{P S\},\{P P, I U\},\{P O, C I\}$ \\
$\{P S\}$ & $\{P S\},\{P P, C I\},\{P O, I U\}$ \\
$\{P S, C I\}$ & $\{P S, C I\},\{P P, P O, I U\}$ \\
$\{P S, P O\}$ & $\{P S, P O\},\{P P, C I, I U\}$ \\
$\{P S, I U\}$ & $\{P S, I U\},\{P P, P O\},\{C I\}$ \\
$\{P P, P O\}$ & $\{P P, P O\},\{P S\},\{C I, I U\}$ \\
$\{P P, P O, C I\}$ & $\{P P, P O, C I\},\{P S, I U\}$ \\
$\{P P, C I, I U\}$ & $\{P P, C I, I U\},\{P S\},\{P O\}$ \\
\hline
\end{tabular}

Table 1: Minimal winning embedded coalitions.

embedded coalitions (sixth and ninth rows of Table 1). Another difference that can be observed from the list above is that $P P, P O$, and $C I$ are not symmetric players anymore.

In Table 2 we depict the Deegan-Packel and Public Good power indices of the parties in the Parliament of Andalusia both when we consider the simple game in characteristic function form (majority rule) and in partition function form (plurality rule).

\begin{tabular}{|c|c|c|c|c|c|}
\cline { 2 - 6 } \multicolumn{2}{c|}{} & \multicolumn{2}{c|}{ Charac. function } & \multicolumn{2}{c|}{ Partition function } \\
\cline { 2 - 6 } & Seats & D-P & P-G & DP & PG \\
\hline \hline$P S$ & 47 & .375 & .33 & .500 & .353 \\
\hline$P P$ & 33 & .208 & .22 & .130 & .176 \\
\hline$P O$ & 15 & .208 & .22 & .148 & .176 \\
\hline$C I$ & 9 & .208 & .22 & .130 & .176 \\
\hline$I U$ & 5 & 0 & 0 & .093 & .118 \\
\hline
\end{tabular}

Table 2: The Deegan-Packel and Public Good indices for the simple games in characteristic and partition function form.

Several comments are in order. First, when we move from the simple majority to the plurality rule, the strongest party becomes more powerful. This can be explained by the fact that with the majority rule $P S$ needs 8 more seats to form a winning coalition. However, when we consider 
the plurality rule depending on how the rest of the players are organized, $P S$ alone can form a winning coalition (see Table 1). Second, the three parties that were symmetric with the simple majority rule decrease their power. This can also be explained by the fact that it is easier to form a winning coalition with the plurality rule than with the majority rule. Therefore, it is more difficult for intermediate parties to be critical, i.e., it is more difficult for them to participate in a minimal winning embedded coalition. Third, as we have already mentioned $I U$ is not a null player when we consider the plurality rule. This party is too small to participate in a minimal winning coalition with the majority rule, but it participates in two minimal winning embedded coalitions. Forth, according to the PG index, $P P, P O$, and $C I$ have the same power. This is surprising given the difference between their seats and because they are not symmetric players. However, it is easy to see from Table 1 that they do participate in the same number of minimal winning embedded coalitions.

Finally, and probably the most surprising observation is that according to the DP index, $P O$ has more power than $P P$ even though it has less seats. In Table 1, we can see that the only difference between $P P$ and $P O$ is in lines 5 and 9 . Since the DP index shares the power equally among the members of the active coalition, $P O$ is being allocated more power than $P P$, even though it has less seats. That is, the DP index does not satisfy local monotonicity. It is easy to find an example to show that the PG index does not satisfy it either. Local monotonicity requires that if player $i$ has less seats than player $j$, then $i$ cannot have more power than $j$. Felsenthal and Machover (1995) propose (in addition to three postulates) local monotonicity as major desiderata for a measure of voting power. Even though the DP and PG power indices do not satisfy local monotonicity, they are interesting measures in situations where minimal winning coalitions play an important role. For instance, Holler and Napel (2005) argue that taking only minimal winning coalitions to measure power is adequate because any other winning coalition contains redundant members.

\section{Conclusions}

Games in partition function form capture the fact that the value of a group of agents may depend on how the others organize themselves. Here, any kind of unordered partition of the agents is possible in contrast with other models. For instance, the partition is fixed in the a priori unions model (Owen, 1977; Hart and Kurz, 1983) and in games with $r$ alternatives there is an order in the elements of the partition (see for instance, Bolger, 1993; Amer et al., 1998; 
Carreras and Magaña, 2008, among others).

In the literature, as we mention in the introduction, several extensions of simple games have been proposed for games in partition function form. Different approaches arise from different ways of extending the binary relation of inclusion to this setting. We introduce a class of simple games in the context of games in partition function form by defining a property of monotonicity related to the one in Carreras and Magaña (2008) in the setting of games with $r$ alternatives and inspired by the basis proposed by de Clippel and Serrano (2008). The main feature of our inclusion relation is that the active coalition grows while the partition of the remaining agents becomes finer.

In our paper, we have defined and characterized two values which are natural extensions of the Deegan-Packel and Public Good indices, proposed and used to measure power in simple games in characteristic function. A real example, using the plurality voting rule as decision procedure, illustrates their applicability and some of their drawbacks. Indeed, these power indices violate local monotonicity, in the same line as their counterparts for simple games in characteristic function. It is easy to see that other non-local monotonic power indices like the Shift power index (Alonso-Meijide and Freixas, 2010) can also be generalized to this framework. The extension of the Banzhaf power index is a topic for future research.

Another interesting issue that remains open is the development of procedures to ease the computation of these indices. Notice that of the number of embedded coalitions grows faster than the number of regular coalitions as the number of agents increases. In relation to these, the indices built considering only minimal winning embedded coalitions can be computed in an easier way once they are identified. Then, it can be interesting to find efficient procedures to obtain this type of embedded coalitions. ${ }^{4}$

\section{Acknowledgment}

This research has been supported by the Spanish Ministerio de Economía y Competitividad under grants MTM2014-53395-C3-2-P, MTM2014-53395-C3-3-P, and ECO2014-52340-P and by Generalitat de Catalunya under grant 2014SGR40.

\footnotetext{
${ }^{4} \mathrm{~A}$ multilinear extension procedure is proposed in Alonso-Meijide et al. (2015).
} 


\section{References}

Alonso-Meijide, J., Casas-Méndez, B., Holler, M., and Lorenzo-Freire, S. (2008). Computing power indices: Multilinear extensions and new characterizations. European Journal of Operational Research, 188:540-554.

Alonso-Meijide, J. M., Álvarez-Mozos, M., and Fiestras-Janeiro, M. G. (2009). Values of games with graph restricted communication and a priori unions. Mathematical Social Sciences, $58: 202-213$.

Alonso-Meijide, J. M., Álvarez-Mozos, M., and Fiestras-Janeiro, M. G. (2015). Power indices and minimal winning coalitions in simple games with externalities. Technical report, UB Economics Working Papers.

Alonso-Meijide, J. M. and Bowles, C. (2005). Generating functions for coalitional power indices: An application to the IMF. Annals of Operations Research, 137:21-44.

Alonso-Meijide, J. M., Casas-Méndez, B., and Fiestras-Janeiro, M. G. (2013). A review of some recent results on power indices. In Power, Voting, and Voting Power: 30 Years After, pages 231-245. Springer Science Business Media.

Alonso-Meijide, J. M. and Freixas, J. (2010). A new power index based on minimal winning coalitions without any surplus. Decision Support Systems, 49(1):70-76.

Álvarez-Mozos, M., Alonso-Meijide, J. M., and Fiestras-Janeiro, M. G. (2016). The ShapleyShubik index in the presence of externalities. Technical report, UB Economics Working Papers.

Álvarez-Mozos, M., Ferreira, F., Alonso-Meijide, J., and Pinto, A. A. (2015). Characterizations of power indices based on null player free winning coalitions. Optimization, 64:675-686.

Álvarez-Mozos, M. and Tejada, O. (2015). The Banzhaf value in the presence of externalities. Social Choice and Welfare, 44:781-805.

Amer, R., Carreras, F., and Magaña, A. (1998). The Banzhaf-Coleman index for games with $\mathrm{r}$ alternatives. Optimization, 44:175-198.

Banzhaf, J. F. (1964). Weighted voting doesn't work: A mathematical analysis. Rutgers Law Review, 19:317. 
Bolger, E. (1983). The Banzhaf index for multicandidate presidential elections. SIAM Journal of Algebraic Discrete Methods, 4:422-458.

Bolger, E. (1986). Power indices for multicandidate voting games. International Journal of Game Theory, 15:175-186.

Bolger, E. (1990). A characterization of an extension of the Banzhaf value for multicandidate voting games. SIAM Journal of Discrete Mathematics, 3:466-477.

Bolger, E. (1993). A value for games with $n$ players and $r$ alternatives. International Journal of Game Theory, 22:319-334.

Carreras, F. and Magaña, A. (2008). The Shapley-Shubik index for simple games with multiple alternatives. Annals of Operations Research, 158:81-97.

de Clippel, G. and Serrano, R. (2008). Marginal contributions and externalities in the value. Econometrica, 76:1413-1436.

Deegan, J. and Packel, E. (1978). A new index of power for simple $n$-person games. International Journal of Game Theory, 7:113-123.

Dutta, B., Ehlers, L., and Kar, A. (2010). Externalities, potential, value and consistency. Journal of Economic Theory, 145:2380-2411.

Felsenthal, D. and Machover, M. (1995). Postulates and paradoxes of relative power indices A critical re-appraisal. Theory and Decision, 38:195-229.

Felsenthal, D. S. and Machover, M. (1998). The Measurement of Voting Power: Theory and Practice, Problems and Paradoxes. Edward Elgar Pub.

Grabisch, M. (2010). The lattice of embedded subsets. Discrete Applied Mathematics, 158:479488.

Hafalir, I. (2007). Efficiency in coalition games with externalities. Games and Economic Behavior, 61:242-258.

Hart, S. and Kurz, M. (1983). Endogenous formation of coalitions. Econometrica, 51:1047-1064.

Holler, M. and Napel, S. (2005). Local monotonicity of power: axiom or just a property? Quality and Quantity, 38:637-647. 
Holler, M. J. (1982). Forming coalitions and measuring voting power. Political Studies, 30:262271.

Holler, M. J. and Packel, E. W. (1983). Power. Luck and the Right Index. Journal of Economics, $43: 21-29$.

Johnston, R. J. (1978). On the measurement of power: Some reactions to laver. Environment and Planning A, 10:907-914.

Lorenzo-Freire, S., Alonso-Meijide, J., Casas-Méndez, B., and Fiestras-Janeiro, M. (2007). Characterizations of the Deegan-Packel and Johnston power indices. European Journal of Operational Research, 177:431-444.

Myerson, R. (1977). Values of games in partition function form. International Journal of Game Theory, 6:23-31.

Owen, G. (1977). Values of games with a priori unions. In Mathematical economics and game theory, pages 76-88. Springer.

Shapley, L. S. and Shubik, M. (1954). A method for evaluating the distribution of power in a committee system. American Political Science Review, 48:787-792.

Thrall, R. and Lucas, W. (1963). n-person games in partition function form. Naval Research Logistics Quarterly, 10:281-298.

von Neumann, J. and Morgenstern, O. (1944). Theory of Games and Economic Behavior. Princeton University Press.

Weber, R. J. (1988). Probabilistic values for games. The Shapley Value. Essays in Honor of Lloyd S. Shapley, pages 101-119. 